\title{
Les stratégies d'apprentissage des apprenants de la première année des filières de français de l'Université du Costa Rica
}

\author{
Alexandra Arrieta Espinoza \\ Escuela de Lenguas Modernas \\ Universidad de Costa Rica \\ KuOK-Wa ChaO ChaO \\ Escuela de Lenguas Modernas \\ Universidad de Costa Rica
}

\begin{abstract}
Résumé
Cet article analyse les résultats d'une recherche sur les stratégies d'apprentissage des apprenants de la première année des filières d'enseignement du français et de français de l'Université du Costa Rica. Pour l'analyse des résultats, nous avons utilisé la typologie d'O'Malley et Chamot (1990) qui classifie les stratégies en trois grandes catégories: cognitives, métacognitives et socio-affectives. Cette recherche présente des caractéristiques d'une étude quantitative.
\end{abstract}

Mots clés: stratégies d'apprentissage, stratégies cognitives, stratégies métacognitives, stratégies socio-affectives, français, L2

\section{Resumen}

Este artículo analiza los resultados de una investigación sobre las estrategias de aprendizaje de los aprendices del primer año de las carreras de Bachillerato en la enseñanza del francés y Bachillerato en francés de la Universidad de Costa Rica. Para el análisis de los resultados, utilizamos la tipología de O'Malley y Chamot (1990) que clasifica las estrategias en tres grandes categorías: cognitivas, metacognitivas y socioafectivas. Esta investigación presenta las características de un estudio de tipo cuantitativo.

Palabras claves: estrategias de aprendizaje, estrategias cognitivas, estrategias metacognitivas, estrategias socioafectivas, francés, L2 


\section{Introduction}

$\mathrm{D}$ ans le cadre du processus d'autoévaluation de la filière de français, nous avons considéré pertinent et nécessaire de connaître les différentes stratégies d'apprentissage employées par les apprenants en ce qui concerne la compréhension et l'expression écrites.

Afin d'analyser ces stratégies, nous proposons une recherche visant à les identifier et à les classifier selon les typologies d'O'Malley et Chamot (1990). Ces auteurs abordent les stratégies d'apprentissage sous les angles métacognitif, cognitif et socio-affectif.

Les résultats obtenus nous permettront de mieux comprendre le processus d'acquisition et d'apprentissage des apprenants de français langue étrangère.

\section{Cadre théorique}

\subsection{Le concept de stratégie d'apprentissage}

Le terme « stratégie d'apprentissage » dans le domaine de l'acquisition des langues est défini par de nombreux auteurs, tels que Wenden (1987) qui donne différents critères pour cerner la notion de stratégies. Donc, pour cet auteur, les stratégies sont des actions ou techniques spécifiques et non pas des traits de personnalité ou de style cognitif. Elles sont orientées vers un problème, contribuent directement ou indirectement à l'apprentissage, sont utilisées consciemment pour l'acquisition ou la communication, et deviennent automatiques, sont négociables, modifiables, apprises ou rejetées.

MacIntyre (1994, p. 190) définit les stratégies comme des actions choisies par les apprenants d'une langue étrangère dans le but de se faciliter les tâches d'acquisition et de communication.

Selon Cyr (1998, p. 4), les stratégies d'apprentissage sont considérées comme des comportements, des techniques, des tactiques, des plans, des opérations mentales conscientes, inconscientes ou potentiellement conscientes, des habiletés cognitives ou fonctionnelles, et aussi des techniques de résolution de problèmes observables chez l'individu qui se trouve en situation d'apprentissage.

Larue et Cossette (2005, p. 26) envisagent les stratégies commeles pensées etcomportements d'un étudiant, qui, engagé dans un apprentissage, utilise des procédures ou des actions spécifiques, facilitant son apprentissage et optimisant sa performance.

Bégin (2008, p. 53) considère les stratégies comme une catégorie d'actions métacognitives ou cognitives utilisées dans une situation d'apprentissage, orientées dans un but de réalisation d'une tâche ou d'une activité scolaire et servant à effectuer des opérations sur les connaissances en fonction d’objectifs précis.

Pour cette recherche, nous concevons les stratégies d'apprentissage comme des actions ou des comportements entrepris par les apprenants afin de faire face à l'acquisition des connaissances en matière d'expression et de compréhension écrites, ainsi que pour atteindre les objectifs d'apprentissage. 


\subsection{La typologie d'O'Malley et Chamot}

O’Malley et Chamot considèrent les stratégies d'apprentissage comme " des pensées ou des comportements spéciaux utilisés par les apprenants pour les aider à comprendre, apprendre, ou à maintenir la nouvelle information " (O'Malley et Chamot, 1990, p.1). Cette typologie est considérée comme la plus synthétique, la plus rigoureuse et la plus solidement ancrée dans le concept de la théorie de la psychologie cognitive, ainsi que plus pratique et plus facile à utiliser pour les enseignants de langue seconde intéressés aux stratégies de leurs apprenants.

Ces auteurs distinguent trois grandes catégories de stratégies d'apprentissage :

a. Stratégies métacognitives

b. Stratégies cognitives

c. Stratégies socio-affectives

\subsubsection{Stratégies métacognitives}

Les stratégies métacognitives (Lafontaine, 2005 ; Larue et Cossette, 2005 ; Lee, 2011 ; Tang, 2011) sont celles qui renvoient à des processus impliqués dans le contrôle conscient des activités cognitives. Ce sont des stratégies de planification qui réunissent des stratégies d'organisation, d'attention et de gestion, d'identification des problèmes, d'autorégulation, de même que des stratégies de contrôle et d'évaluation.

Selon Cyr (1998), les stratégies métacognitives peuvent être classées de la manière suivante:

a. L'anticipation ou la planification. Elle consiste à fixer des buts à court ou à long terme; à étudier par soi-même un point de langue ou un thème qui n’a pas encore été abordé en salle de classe; à prévoir les éléments linguistiques nécessaires à l'accomplissement d'une tâche d'apprentissage ou d'un acte de communication.

b. L'attention. Elle consiste à prêter attention à tout intrant langagier susceptible de contribuer à l'apprentissage; à maintenir son attention au cours de l'exécution d'une tâche.

c. L'autogestion. Elle consiste à comprendre les conditions qui facilitent l'apprentissage de la langue et à chercher à réunir ces conditions.

d. L'autorégulation. Elle consiste à vérifier et à corriger sa performance au cours d'une tâche d'apprentissage ou d'un acte de communication.

e. L'identification du problème. Elle consiste à cerner le point central d'une tâche langagière ou un aspect de cette tâche qui nécessite une solution en vue d'une réalisation satisfaisante.

f. L'autoévaluation. Elle consiste à évaluer ses habiletés à accomplir une tâche langagière ou un acte de communication; à évaluer le résultat de sa performance langagière ou de ses apprentissages. 


\subsubsection{Stratégies cognitives}

Les stratégies cognitives (Lafontaine, 2005 ; Larue et Cossette, 2005 ; Lee, 2011 ; Tang, 2011) sont des démarches ou des opérations mentales par lesquelles les apprenants traitent les données linguistiques et sociolinguistiques. Elles concernent les processus d'enregistrement des informations, de conservation des informations en mémoire et de rappel au moment opportun en tenant compte du contexte. Elles regroupent des stratégies de gestion de ressources, de résumé et de prise de notes, de compréhension et de mémorisation. La mémorisation inclut divers types de stratégies : répétition, regroupement, association, recours à l'imagerie, représentation auditive, et enfin, élaboration.

D’après Cyr (1998), les stratégies cognitives peuvent être classées de la manière suivante:

a. La répétition ou la pratique de la langue. Elle consiste à saisir les occasions qui sont offertes de communiquer dans la langue cible; à répéter des segments de la langue; à penser ou se parler à soi-même dans la langue cible; à tester ou réutiliser dans des communications authentiques des mots, des phrases ou des règles appris en salle de classe.

b. La mémorisation. Elle consiste à appliquer diverses techniques mnémoniques.

c. La prise de notes. Elle consiste à noter dans un cahier les mots nouveaux, les concepts, les expressions, les locutions figées ou ritualisées qui peuvent servir à la réalisation d'une tâche d'apprentissage ou d'un acte de communication.

d. Le regroupement. Il consiste à ordonner, à classer ou à étiqueter la matière enseignée selon des attributs communs de manière à faciliter sa récupération.

e. La révision. Elle consiste à surapprendre et à se familiariser à tel point avec la matière que sa récupération devient naturelle et automatique.

f. L'inférence. Elle consiste à utiliser les éléments connus d'un texte ou d'un énoncé afin d'induire ou d'inférer le sens des éléments nouveaux ou inconnus; à utiliser le contexte langagier ou extralangagier dans le but de suppléer aux lacunes dans la maîtrise du code linguistique et afin de comprendre le sens ou la signification globale d'un texte ou d'un acte de communication; à recourir à son intuition pour deviner intelligemment.

g. La déduction. Elle consiste à appliquer une règle réelle ou hypothétique en vue de produire ou de comprendre la L2.

h. La recherche documentaire. Elle consiste à utiliser les sources de référence au sujet de la langue cible.

i. La traduction et la comparaison avec la L1 ou avec une autre langue connue. Elles consistent à traduire ou à faire des comparaisons interlinguales significatives; à se servir de la compétence langagière acquise en L1 ou par l'apprentissage d'une autre L2 afin de comprendre le système et le fonctionnement de la langue cible. 
j. La paraphrase. Elle consiste à suppléer au manque de vocabulaire par la circonlocution en cas d'incompréhension de la part de l'interlocuteur en choisissant d'autres mots ou d'autres phrase de la langue cible pour réaliser l'acte de communication.

k. L'élaboration. Elle consiste à établir des liens entre les éléments nouveaux et les connaissances antérieures; à faire des associations intralinguales dans le but de comprendre ou de produire des énoncés.

1. Le résumé. Il consiste à faire un résumé mental ou écrit d'une règle ou d'une information présentée dans une tâche.

\subsubsection{Stratégies socio-affectives}

Les stratégies socio-affectives (Lafontaine, 2005 ; Larue et Cossette, 2005 ; Lee, 2011 ; Tang, 2011) se rapportent aux activités effectuées par les apprenants en vue de stimuler des dispositions affectives propices à l'apprentissage et de maîtriser le mieux possible les émotions et les sentiments qui pourraient nuire à la concentration ou à la motivation. Elles renvoient aussi aux modes d'interaction des apprenants avec d'autres apprenants et avec des locuteurs natifs en vue de faciliter l'appropriation de la L2. Elles comprennent des stratégies de coopération, de clarification et de stratégies affectives, telles qu'activer des émotions, des croyances et des attitudes de soutien et générer et maintenir la motivation.

Selon Cyr (1998), les stratégies socio-affectives peuvent être classées de la manière suivante:

a. La clarification et la vérification. Elle consiste à demander de répéter; à solliciter auprès du professeur ou d'un locuteur natif des clarifications, des explications ou des reformulations.

b. La coopération. Elle consiste à interagir avec ses pairs dans le but d'accomplir une tâche ou de résoudre un problème d'apprentissage; à solliciter de la part d'un locuteur compétent des appréciations au sujet de sa performance ou de son apprentissage.

c. La gestions des émotions ou la réduction de l'anxiété. Elle consiste à prendre conscience de la dimension affective entourant l'apprentissage d'une L2; à se parler à soi-même en vue de réduire le stress accompagnant l'accomplissement d'une tâche d'apprentissage ou d'un acte de communication; à utiliser diverses techniques qui contribuent à la confiance en soi et à la motivation; à s'encourager et se récompenser; à ne pas craindre de faire des erreurs ou de prendre des risques.

d. L'autorenforcement. Elle consiste à augmenter et à maintenir son propre comportement. 


\section{Méthodologie}

Cette recherche a des caractéristiques d'une étude quantitative, car nous examinons un groupe de 61 apprenants de la première année des filières de français et d'enseignement du français et cet échantillonnage nous permettra d'obtenir des données représentatives quant aux stratégies d'apprentissage dans le domaine de l'expression et de la compréhension écrites.

Les participants de cette recherche présentent les caractéristiques suivantes (voir annexe 1):

Tableau 1

Caractéristiques principales des participants

\begin{tabular}{ll}
\hline \multicolumn{1}{c}{ Caractéristiques } & \multicolumn{1}{c}{ Pourcentage } \\
\hline Genre & 14 hommes $(22,95 \%)$ \\
& 47 femmes $(77,04 \%)$ \\
Age & 18 à $20-39$ personnes $(63,93 \%)$ \\
& 21 à $25-13$ personnes $(21,31 \%)$ \\
& 26 à $30-3$ personnes $(4,91 \%)$ \\
& plus de 31 - 5 personnes $(8,19 \%)$ \\
& 1 personne sans réponse $(1,63 \%)$ \\
& célibataires - 58 personnes $(95,08 \%)$ \\
Etat civil & mariés - 2 personnes $(3,27 \%)$ \\
& divorcés - 1 personne $(1,63 \%)$ \\
Lieu de provenance & San José - 36 personnes $(59,01 \%)$ \\
& Heredia - 9 personnes $(14,75 \%)$ \\
& Alajuela - 4 personnes $(6,55 \%)$ \\
Type d'établissement & Cartago - 12 personnes $(19,67 \%)$ \\
d'études secondaires & public - 36 personnes $(59,01 \%)$ \\
& semi-privé - 11 personnes $(18,03 \%)$ \\
Filières & privé - 14 personnes $(22,95 \%)$ \\
& Licence en français - 43 personnes $(70,49 \%)$ \\
& Licence en enseignement du français - 17 personnes $(27,86 \%)$ \\
Maîtrise d'autres & 1 personne sans réponse $(1,63 \%)$ \\
langues étrangères & Oui - 35 personnes $(57,37 \%)$ \\
& Non - 25 personnes $(40,98 \%)$ \\
& 1 personne sans réponse $(1,63 \%)$ \\
\hline
\end{tabular}

Le corpus de cette recherche est composé d'un instrument (voir annexe 1) répondu par les apprenants pendant le deuxième cycle de l'année 2013. L'instrument comporte une série de questions se rapportant aux stratégies cognitives, métacognitives et socio-affectives dans le domaine de l'expression et de la compréhension écrites.

Le questionnaire que nous avons utilisé a été pris de Cyr (1998), Harris (2002) et Boudon (2006) et comporte quelques petites modifications plus adaptées 
á notre contexte costaricien . Ce questionnaire comprend trois grandes parties, chacune consacrée à un type de stratégie : cognitive, métacognitive et socio-affective. La partie des stratégies cognitives est divisée en quatre domaines : compréhension écrite, apprentissage du vocabulaire, apprentissage des règles grammaticales et écriture.

Pour cette étude, nous avons suivi plusieurs étapes: la conception de l'instrument, l'application de celui-ci aux apprenants de la première année, le regroupement des données et l'analyse des résultats.

\section{Analyse des résultats}

Pour la compréhension des résultats, nous aborderons premièrement les stratégies cognitives par rapport à la compréhension écrite, à la grammaire, au lexique et à la production écrite. Ensuite, nous procèderons à l'analyse des stratégies métacognitives, et finalement, des stratégies socio-affectives.

\subsection{Les stratégies cognitives}

Les stratégies cognitives impliquent une interaction avec la matière en étude, une manipulation mentale ou physique de celle-ci et une application de techniques spécifiques dans l'exécution d'une tâche d'apprentissage.

Les apprenants de la première année des filières de français et de l'enseignement du français se servent de différentes stratégies cognitives pour la compréhension écrite, tel qu'il est illustré au tableau 2:

Tableau 2

Les stratégies de compréhension écrite

\begin{tabular}{|c|c|c|c|c|}
\hline Stratégies & Jamais & $\begin{array}{c}\text { Quelque- } \\
\text { fois }\end{array}$ & Souvent & $\begin{array}{c}\text { Sans } \\
\text { réponse }\end{array}$ \\
\hline $\begin{array}{l}\text { 1. J'examine la typographie (le titre, } \\
\text { les images, etc.) du document afin } \\
\text { de déterminer le type de texte, par } \\
\text { exemple : lettre, poème, article, etc. }\end{array}$ & $\begin{array}{c}5 \\
(8,20 \%)\end{array}$ & $\begin{array}{c}22 \\
(36,07 \%)\end{array}$ & $\begin{array}{c}34 \\
(55,74 \%)\end{array}$ & \\
\hline $\begin{array}{l}\text { 2. Je regarde attentivement les images } \\
\text { et le titre pour pouvoir déduire le su- } \\
\text { jet en question. }\end{array}$ & & $\begin{array}{c}23 \\
(37,70 \%)\end{array}$ & $\begin{array}{c}38 \\
(62,30 \%)\end{array}$ & \\
\hline $\begin{array}{l}\text { 3. J'essaie de me remémorer autant de } \\
\text { mots que possible en rapport avec le } \\
\text { sujet abordé. }\end{array}$ & & $\begin{array}{c}36 \\
(59,02 \%)\end{array}$ & $\begin{array}{c}24 \\
(39,34 \%)\end{array}$ & $\begin{array}{c}1 \\
(1,64 \%)\end{array}$ \\
\hline $\begin{array}{l}\text { 4. Je lis la totalité du texte afin d'en } \\
\text { saisir l'idée générale. }\end{array}$ & $\begin{array}{c}1 \\
(1,64 \%)\end{array}$ & $\begin{array}{c}8 \\
(13,11 \%)\end{array}$ & $\begin{array}{c}52 \\
(85,25 \%)\end{array}$ & \\
\hline
\end{tabular}


5. Je repère les mots apparentés ou qui me sont familiers.

6. J'identifie les termes qui me paraissent être des mots-clés.

7. Je m'appuie sur mes connaissances acquises pour en déduire des hypothèses plausibles.

8. J'essaie de tirer des indices à partir de la ponctuation.

9. Je prononce à haute voix les mots qui ne me sont pas familiers dans le but de mieux les comprendre.

10. Je lis le texte à haute voix pour repérer la composition des phrases et les parties sur lesquelles je dois me concentrer les unes après les autres.

11. Je substitue les mots que je ne connais pas par d'autres mots dans ma langue maternelle.

12. Je décompose les mots qui ne me sont pas familiers pour associer certains éléments à des mots que je connais.

13. Je repère les mots nouveaux et je cherche leur sens dans le dictionnaire 14. Je regarde attentivement la terminaison des verbes pour en déterminer le temps et identifier le sujet.

15. Je fais un résumé de la lecture.
$2(3,28 \%)$

$$
\begin{gathered}
1 \\
(1,64 \%) \\
2
\end{gathered}
$$$$
(3,28 \%)
$$

$11(18,03 \%)$

$7(11,48 \%)$

5

$(8,20 \%)$

6

$(9,84 \%)$

$7(11,48 \%)$

$2(3,28 \%)$

$25(40,98 \%)$
(37,70\%)

$(32,79 \%)$

19

(31,15\%)

24

$(39,34 \%)$

24

$(39,34 \%)$

30

$(49,18 \%)$

$(36,07 \%)$

$(44,26 \%)$

$(45,90 \%)$
23

20

22

27

28

27

$(44,26 \%)$

20

$(32,79 \%)$

36

$(59,02 \%)$

41

$(67,21 \%)$

23

11

\section{2}

29

$(47,54 \%)$

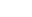

$55,74 \%$

$37.70 \%)$
1

(1,64\%)

1

$(1,64 \%)$

35

$57,38 \%)$

19

1

$1,64 \%)$
2

$(3,28 \%)$

De cette façon, nous pouvons observer que plus d'un 50\% d'apprenants utilise souvent des stratégies cognitives d'inférence, telles que les stratégies 1, 2, 4 et 5 . Ils emploient quelquefois, autour d'un 50\%, des stratégies d'inférence, telles que les stratégies 8 et 12 . Cependant, nous remarquons que pour ces mêmes stratégies 8 et 12, moins d'un $20 \%$ ne s'en sert jamais.

Nous retrouvons aussi que plus d'un $60 \%$ utilise souvent la stratégie de déduction proposée dans le cas 14. Plus d'un 30\% l'emploie quelquefois.

La majorité des apprenants utilise souvent la stratégie d'inférence et déduction 7 et moins d'un $40 \%$ l'emploie quelquefois.

Plus d'un $45 \%$ emploie souvent les stratégies de répétition 9 et 10 . Moins d'un $50 \%$ utilise quelquefois ces mêmes stratégies et autour d'un 10\% ne les utilise jamais.

Pour ce qui est la stratégie de regroupement 3 , nous apercevons que moins d'un 40\% l'utilise souvent contre plus d'un 50\% qui l'emploie quelquefois.

Quant à la stratégie de prise de notes 6, plus d'un 50\% l'emploie souvent, moins d'un $40 \%$ l'utilise quelquefois et très peu d'étudiants ne l'emploient jamais. 
Pour la stratégie de recherche documentaire 13, presque le 60\% l'utilise souvent, moins d'un 40\% l'emploie quelquefois et moins d'un $5 \%$ ne l'utilise jamais.

En ce qui concerne la stratégie de traduction 11, autour d'un 45\% l'utilise souvent ou quelquefois, mais presqu'un 10\% ne l'emploie jamais.

Il est intéressant de remarquer que la stratégie de résumé 15 n'est jamais utilisée par un 40\% d'apprenants. Elle est utilisée quelquefois par moins d'un $40 \%$ et souvent par un $18 \%$.

Selon les résultats obtenus, nous remarquons que les apprenants se servent majoritairement des stratégies d'inférence, de déduction, de prise de notes et de recherche documentaire, ce qui montre une activité mentale consciente dans le but de trouver un sens à la nouvelle langue en cours d'acquisition. L'apprenant essaie de saisir les données afin de comprendre le fonctionnement de la langue ou le sens global d'une lecture. Quoiqu'ils utilisent ces types de stratégies, nous pouvons noter que la répétition est encore souvent utilisée par un pourcentage important des apprenants, ce qui traduit le désir de pratiquer la langue et de fixer les nouvelles structures linguistiques. La stratégie de traduction est encore souvent employée, attitude justifiée par le fait que les débutants font recours généralement à leur langue maternelle. Toutefois, il est intéressant de signaler qu'un pourcentage élevé d'apprenants n'utilise jamais la stratégie de résumé, ce qui obéit essentiellement à leur manque de maturité linguistique, car il s'agit des étudiants débutants.

Le tableau 3 montre les stratégies d'apprentissage du vocabulaire:

Tableau 3

Les stratégies d'apprentissage du vocabulaire

\begin{tabular}{|c|c|c|c|c|}
\hline Stratégies & Jamais & $\begin{array}{l}\text { Quelque- } \\
\text { fois }\end{array}$ & Souvent & $\begin{array}{l}\text { Sans } \\
\text { réponse }\end{array}$ \\
\hline $\begin{array}{l}\text { 1. J'associe un mot que j'essaie d'ap- } \\
\text { prendre à un mot similaire dans ma } \\
\text { langue maternelle. }\end{array}$ & $\begin{array}{c}3 \\
(4,92 \%)\end{array}$ & $\begin{array}{c}23 \\
(37,70 \%)\end{array}$ & $\begin{array}{c}35 \\
(57,38 \%)\end{array}$ & \\
\hline 2. J'en fais un dessin. & $\begin{array}{c}26 \\
(42,62 \%)\end{array}$ & $\begin{array}{c}23 \\
(37,70 \%)\end{array}$ & $\begin{array}{c}12 \\
(19,67 \%)\end{array}$ & \\
\hline $\begin{array}{l}\text { 3. J'écris les mots plusieurs fois de } \\
\text { suite. }\end{array}$ & $\begin{array}{c}16 \\
(26,23 \%)\end{array}$ & $\begin{array}{c}23 \\
(37,70 \%)\end{array}$ & $\begin{array}{c}22 \\
(36,07 \%)\end{array}$ & \\
\hline $\begin{array}{l}\text { 4. J'utilise la technique « lire-cacher- } \\
\text { tester-vérifier ». }\end{array}$ & $\begin{array}{c}22 \\
(36,07 \%)\end{array}$ & $\begin{array}{c}22 \\
(36,07 \%)\end{array}$ & $\begin{array}{c}17 \\
27,87 \%)\end{array}$ & \\
\hline $\begin{array}{l}\text { 5. Je regarde le mot, puis je ferme les } \\
\text { yeux et j'essaie de me le représenter } \\
\text { dans mon esprit (mémoire photogra- } \\
\text { phique). }\end{array}$ & $\begin{array}{c}21 \\
(34,43 \%)\end{array}$ & $\begin{array}{c}18 \\
(29,51 \%)\end{array}$ & $\begin{array}{c}22 \\
(36,07 \%)\end{array}$ & \\
\hline 6. Je décompose les mots longs. & $16(26,23 \%)$ & $35(57,38 \%)$ & $10(16,39 \%)$ & \\
\hline $\begin{array}{l}\text { 7. J'utilise les mots pour en faire un } \\
\text { poème de toute sorte. }\end{array}$ & $\begin{array}{c}48 \\
(78,69 \%)\end{array}$ & $\begin{array}{c}7 \\
(11,48 \%)\end{array}$ & $\begin{array}{c}6 \\
(9,84 \%)\end{array}$ & \\
\hline
\end{tabular}


8. Je mets tout autour de ma chambre des notes autocollantes sur lesquelles j'ai inscrit les mots à apprendre.

9. Je crée deux jeux de cartes - l'un contenant les mots en langue étrangère, l'autre les images ou les mots correspondants dans ma langue maternelle afin d'apprendre de façon ludique.

10. Je sépare les mots de leurs articles et j'essaie ensuite de les rapparier.

11. Je fais une recherche sur le mot et je l'utilise dans des phrases.

12. Je crée un exercice à trous en y intégrant les mots nouveaux.

13. Je prononce les mots à voix haute plusieurs fois de suite.

15. Je fais des exercices en ligne (exercices à trous, question à choix multiple, etc.) pour assimiler le vocabulaire.

16. Je regroupe les mots en familles.

17. Je repère dans des documents (écrits, sonores, visuels) le vocabulaire étudié pour le comprendre en contexte afin de le fixer dans ma mémoire.
32

$(52,46 \%)$

42

$(68,85 \%)$

36

$(59,02 \%)$

8

$(13,11 \%)$

33

$(54,10 \%)$

3

$(4,92 \%)$

18

$(29,51 \%)$

23

$(37,70 \%)$

9

$(14,75 \%)$
16

$(26,23 \%)$

17

$(27,87 \%)$
13

$(21,31 \%)$

2

$(3,28 \%)$

18. Je crée un réseau d'associations.

$37(60,66 \%) \quad 15(24,59 \%) \quad 9(14,75 \%)$

Nous pouvons constater que les stratégies cognitives de mémorisation 2,3 , 4, 5, 8 et 10 sont moins utilisées par les apprenants de la première année. Ainsi, pour les cas 2, 8 et 10, plus d'un 40\% ne les emploie jamais. Cependant, pour la 6 , nous remarquons que plus d'un 50\% l'utilise quelquefois.

Par rapport aux stratégies de répétition, l'énoncé 7 est rarement utilisé par ces apprenants dont 78,69\% affirme ne jamais l'employer; contrairement à la stratégie 13 qui est souvent utilisée par 54,10\% et quelquefois par 40,18\%. La stratégie 15 est quelquefois employée par presqu'un 50\% des apprenants. Presqu'un 30\% affirme ne jamais l'utiliser contre un $20 \%$ qui l'utilise souvent.

Pour la stratégie de traduction, nous pouvons contraster l'énoncé 1 et l'énoncé 9, car nous remarquons que pour le premier, 57,38\% l'utilise souvent et $37,70 \%$ quelquefois tandis que pour le deuxième, 68,85\% ne l'emploie jamais et autour d'un 30\% l'utilise souvent ou quelquefois.

Quant au regroupement, nous remarquons que pour la stratégie 18, 60\% ne l'utilise jamais, moins d'un $25 \%$ quelquefois et moins d'un $15 \%$ souvent tandis que pour la stratégie 16, moins d'un 40\% affirme ne jamais l'utiliser ou l'utiliser quelquefois.

Par rapport à la stratégie de recherche documentaire et élaboration 11, presqu'un 50\% l'utilise quelquefois et plus d'un 30\% souvent. 
La stratégie d'élaboration 12, n'est jamais utilisée par plus d'un 50\%.

En ce qui concerne la stratégie d'inférence 17, elle est souvent utilisée par $50 \%$ et plus d'un $30 \%$ quelquefois.

Afin de synthétiser les données concernant les stratégies cognitives pour l'apprentissage du vocabulaire, nous observons que les stratégies de mémorisation, de traduction, de regroupement et de répétition proposées dans le questionnaire ne sont pas très appréciées par les apprenants, car un nombre important d'apprenants affirme ne jamais les utiliser, ceci dû probablement à des aspects culturels où les activités ludiques pour mémoriser le nouveau lexique sont rarement appliquées par les apprenants costariciens. Cependant, il faudrait remarquer que les stratégies de traduction et de répétition n'impliquant pas l'aspect ludique sont généralement employées par les apprenants, ainsi que les stratégies d'inférence et de recherche documentaire.

Dans le tableau 4, nous pouvons apprécier les stratégies d'apprentissage des règles grammaticales.

Tableau 4

Les stratégies d'apprentissage des règles grammaticales

\begin{tabular}{|c|c|c|c|c|}
\hline Stratégies & Jamais & $\begin{array}{l}\text { Quelque- } \\
\text { fois }\end{array}$ & Souvent & $\begin{array}{l}\text { Sans } \\
\text { réponse }\end{array}$ \\
\hline 1. Je mets les règles en musique. & $\begin{array}{c}45 \\
(73,77 \%)\end{array}$ & $\begin{array}{c}11 \\
(18,03 \%)\end{array}$ & $\begin{array}{c}5 \\
(8,20 \%)\end{array}$ & \\
\hline $\begin{array}{l}\text { 2. Je fais des jeux, par exemple : clas- } \\
\text { ser les différents groupes de verbes. }\end{array}$ & $\begin{array}{c}24 \\
(39,34 \%)\end{array}$ & $\begin{array}{c}22 \\
36,07 \%)\end{array}$ & $\begin{array}{c}15 \\
(24,59 \%)\end{array}$ & \\
\hline $\begin{array}{l}\text { 3. Je crée un mot en utilisant les pre- } \\
\text { mières lettres. }\end{array}$ & $\begin{array}{c}12 \\
(19,67 \%)\end{array}$ & $\begin{array}{c}22 \\
(36,07 \%)\end{array}$ & $\begin{array}{c}27 \\
(44,26 \%)\end{array}$ & \\
\hline $\begin{array}{l}\text { 4. Je mets tout autour de ma chambre } \\
\text { des notes autocollantes sur lesquelles } \\
\text { j'ai inscrit les verbes ou les règles } \\
\text { grammaticales à apprendre. }\end{array}$ & $\begin{array}{c}3 \\
(52,46 \%)\end{array}$ & $\begin{array}{c}16 \\
(26,23 \%)\end{array}$ & $\begin{array}{c}13 \\
(21,31 \%)\end{array}$ & \\
\hline 5. Je crée un réseau d'associations. & $\begin{array}{c}23 \\
(37,70 \%)\end{array}$ & $\begin{array}{c}21 \\
(34,43 \%)\end{array}$ & $\begin{array}{c}15 \\
(24,59 \%)\end{array}$ & $\begin{array}{c}2 \\
(3,28 \%)\end{array}$ \\
\hline $\begin{array}{l}\text { 6. Je me base sur les similitudes et } \\
\text { les différences qui existent avec ma } \\
\text { langue maternelle. }\end{array}$ & $\begin{array}{c}2 \\
(3,28 \%)\end{array}$ & $\begin{array}{c}24 \\
(39,34 \%)\end{array}$ & $\begin{array}{c}35 \\
(57,38 \%)\end{array}$ & \\
\hline $\begin{array}{l}\text { 7. Je suis attentif à la présence de } \\
\text { règles, par exemple : je souligne ou en- } \\
\text { toure les exemples correspondant à une } \\
\text { règle grammaticale dans un document. }\end{array}$ & $\begin{array}{c}4 \\
(6,56 \%)\end{array}$ & $\begin{array}{c}22 \\
(36,07 \%)\end{array}$ & $\begin{array}{c}34 \\
(55,74 \%)\end{array}$ & $\begin{array}{c}1 \\
(1,64 \%)\end{array}$ \\
\hline 8. J'écris plusieurs fois les règles. & $\begin{array}{c}14 \\
(22,95 \%)\end{array}$ & $\begin{array}{c}29( \\
47,54 \%)\end{array}$ & $\begin{array}{c}18 \\
(29,51 \%)\end{array}$ & \\
\hline 9. Je répète oralement les règles. & $\begin{array}{c}15 \\
(24,59 \%)\end{array}$ & $\begin{array}{c}31 \\
(50,82 \%)\end{array}$ & $\begin{array}{c}15 \\
(24,59 \%)\end{array}$ & \\
\hline
\end{tabular}


Pour les stratégies de mémorisation proposées, nous apercevons que les énoncés 1 et 4 ne sont jamais utilisés par plus d'un 50\% des apprenants. Les stratégies 2 et 3, sont quelquefois employées par plus d'un 35\%. Cependant, la stratégie 2 n'est jamais utilisée par presqu'un 40\% tandis que la stratégie 3, elle est souvent employée par un 44,26\%.

En ce qui concerne les stratégies de répétition 8 et 9 , nous pouvons affirmer qu'autour d'un 50\% les utilise quelquefois.

La stratégie de regroupement 5 n'est jamais utilisée par plus d'un 35\% et plus d'un $30 \%$ affirme l'utiliser quelquefois.

La stratégie de traduction 6 est employée par plus d'un 55\% et quelquefois par presqu'un $40 \%$.

La stratégie d'inférence 7 est souvent utilisée par 55\% des apprenants et quelquefois employée par plus d'un 35\%.

Pour résumer, il est pertinent de dire que la plupart des apprenants utilisent des stratégies cognitives d'inférence, de répétition et de traduction. Ceci montre probablement un intérêt de la part des apprenants pour pratiquer la langue, essayer de découvrir son fonctionnement et comparer la langue cible à la langue maternelle. Néanmoins, il est important de remarquer qu'un grand groupe d'apprenants n'utilise jamais les stratégies de mémorisation, surtout des stratégies associées à l'aspect ludique pour l'apprentissage des règles d'une langue. Il faudrait aussi dire qu'un nombre important d'apprenants n'emploie pas de stratégies de regroupement et de répétition.

Le tableau 5 illustre les différentes stratégies d'écriture employées par les apprenants de la première année:

Tableau 5

Les stratégies d'écriture

\begin{tabular}{|c|c|c|c|c|}
\hline Stratégies & Jamais & Quelquefois & Souvent & $\begin{array}{c}\text { Sans } \\
\text { réponse }\end{array}$ \\
\hline $\begin{array}{l}\text { 1. Je réfléchis aux exigences posées } \\
\text { par tâches et rassemble toutes les } \\
\text { idées que je veux exprimer. }\end{array}$ & $\begin{array}{c}6 \\
(9,84 \%)\end{array}$ & $\begin{array}{c}37 \\
(60,66 \%)\end{array}$ & $\begin{array}{c}18 \\
(29,51 \%)\end{array}$ & \\
\hline $\begin{array}{l}\text { 2. Je réunis des informations supplémen- } \\
\text { taires en lisant, en parlant avec d'autres } \\
\text { personnes et en me remémorant les } \\
\text { mots et expressions acquis que je pour- } \\
\text { rais utiliser dans le cadre de cette tâche. }\end{array}$ & $\begin{array}{c}13 \\
(21,31 \%)\end{array}$ & $\begin{array}{c}32 \\
(52,46 \%)\end{array}$ & $\begin{array}{c}15 \\
(24,59 \%)\end{array}$ & $\begin{array}{c}1 \\
(1,64 \%)\end{array}$ \\
\hline 3. Je dresse une liste d'idées clés. & $\begin{array}{c}15 \\
(24,59 \%)\end{array}$ & $\begin{array}{c}27 \\
(44,26 \%)\end{array}$ & $\begin{array}{c}19 \\
(31,15 \%)\end{array}$ & \\
\hline $\begin{array}{l}\text { 4. Je choisis la façon d'organiser et de } \\
\text { communiquer mes idées (hiérarchi- } \\
\text { sation, classement, argumentation, } \\
\text { exemples). }\end{array}$ & $\begin{array}{c}10 \\
(16,39 \%)\end{array}$ & $\begin{array}{c}32 \\
(52,46 \%)\end{array}$ & $\begin{array}{c}19 \\
(31,15 \%)\end{array}$ & \\
\hline
\end{tabular}


5. Je fais un plan de travail pour indiquer l'ordre de présentation des idées.

6. Je suis le plan établi préalablement.

7. Je rajoute des idées au fur et à mesure que je rédige.

8. Je consulte le dictionnaire lorsque je ne connais pas un mot ou une expression dont $\mathrm{j}$ 'ai besoin.

9. J'essaie de trouver une autre façon d'exprimer le mot ou l'expression que je ne connais pas.

10. J'essaie d'être aussi précis que possible en employant l'adjectif, le verbe, le nom exacts.

11. J'utilise des connecteurs précis tels que « tout d'abord, puis, ensuite, enfin, etc. ».

12. J'essaie d'employer un style plus élaboré en recourant au lexique approprié. 13. J'essaie d'employer un style plus élaboré en faisant appel aux règles de grammaire que j'ai apprises.

14. Je veille à être précis.

15. Je rédige la version finale.

$14 \quad 26 \quad 21$

$\begin{array}{cccc}(22,95 \%) & (42,62 \%) & (34,43 \%) & \\ 9 & 36 & 15 & 1 \\ (14,75 \%) & (59,02 \%) & (24,59 \%) & (1,64 \%) \\ 9( & 29 & 21 & 2 \\ 14,75 \%) & (47,54 \%) & (34,43 \%) & (3,28 \%) \\ 1 & 12 & 48 & \\ (1,64 \%) & (19,67 \%) & (78,69 \%) & \end{array}$

$\begin{array}{lll}1 & 22 & 38\end{array}$

$(1,64 \%) \quad(36,07 \%) \quad(62,30 \%)$

$\begin{array}{lll}1 & 33 & 27\end{array}$

$(1,64 \%) \quad(54,10 \%) \quad(44,26 \%)$

$\begin{array}{lll}2 & 28 & 31\end{array}$

$(3,28 \%) \quad(45,90 \%) \quad(50,82 \%)$

$\begin{array}{lll}8 & 34 & 19\end{array}$

$(13,11 \%) \quad(55,74 \%) \quad(31,15 \%)$

$\begin{array}{lll}8 & 30 & 23\end{array}$

$(13,11 \%) \quad(49,18 \%) \quad(37,70 \%)$

$\begin{array}{llll}2 & 29 & 28 & 2\end{array}$

$(3,28 \%) \quad(47,54 \%) \quad(45,90 \%) \quad(3,28 \%)$

$\begin{array}{lll}6 & 25 & 30\end{array}$

$(9,84 \%) \quad(40,98 \%) \quad(49,18 \%)$

Pour les stratégies d'élaboration 1, 5, 6 et 15, nous pouvons constater que la plupart les utilise quelquefois. Néanmoins, pour la stratégie 5, plus d'un 20\% affirme ne jamais l'utiliser.

En ce qui concerne les stratégies de répétition 2,7 et 11, nous apercevons que la majorité des étudiants les utilisent quelquefois. La stratégie 2 n'est jamais utilisée par plus d'un 20\% et la stratégie 11 est souvent employée par plus d'un 50\%.

Quant aux stratégies de déduction 10, 13 et 14, elles sont quelquefois utilisées par la plupart des apprenants.

La stratégie de prise de notes 3, est aussi employée quelquefois par les apprenants. Cependant, presqu'un $25 \%$ ne l'utilise jamais.

La stratégie de regroupement 4 est utilisée quelquefois par plus d'un 50\%.

La stratégie de recherche documentaire 8 est souvent employée par plus d'un $70 \%$.

Finalement, la stratégie de paraphrase 9 est souvent employée par plus d'un $60 \%$ des apprenants.

D'après les résultats obtenus du tableau 5, nous notons que les stratégies de déduction, d'élaboration, de paraphrase, de prise de notes, de recherche 
documentaire, de regroupement et de répétition sont très utilisées pour la production écrite par ces apprenants. Cette situation nous fait penser que ces étudiants sont en train d'appliquer les techniques apprises dans leur cours d'expression écrite et de mettre en pratique ces connaissances sur la langue pour pouvoir faire des productions écrites.

\subsection{Les stratégies métacognitives}

Les stratégies métacognitives impliquent une réflexion sur le processus d'apprentissage, une préparation en vue d'apprentissage, le contrôle des activités d'apprentissage, ainsi que l'évaluation et l'autoévaluation.

Tableau 6

Stratégies métacognitives

\begin{tabular}{|c|c|c|c|c|}
\hline Stratégies & Jamais & Quelquefois & Souvent & $\begin{array}{c}\text { Sans } \\
\text { réponse }\end{array}$ \\
\hline $\begin{array}{l}\text { 1. Je lis la totalité du texte pour voir } \\
\text { s'il est cohérent. }\end{array}$ & $\begin{array}{c}2 \\
(3,28 \%)\end{array}$ & $\begin{array}{c}8 \\
(13,11 \%)\end{array}$ & $\begin{array}{c}51 \\
(83,61 \%)\end{array}$ & \\
\hline $\begin{array}{l}\text { 2. Je lis séparément chacun des mots } \\
\text { pour voir s'ils ont l'air correct. }\end{array}$ & $\begin{array}{c}10 \\
(16,39 \%)\end{array}$ & $\begin{array}{c}24 \\
(39,34 \%)\end{array}$ & $\begin{array}{c}27 \\
(44,26 \%)\end{array}$ & \\
\hline $\begin{array}{l}\text { 3. Je prononce les mots pour moi- } \\
\text { même afin de voir s'ils sonnent bien. }\end{array}$ & $\begin{array}{c}5 \\
(8,20 \%)\end{array}$ & $\begin{array}{c}14 \\
(22,95 \%)\end{array}$ & $\begin{array}{c}42 \\
(68,85 \%)\end{array}$ & \\
\hline $\begin{array}{l}\text { 4. Je relis mon travail en faisant attention } \\
\text { à mes fautes habituelles de grammaire } \\
\text { telles que : l'accord des adjectifs, des } \\
\text { verbes; les conjugaisons des verbes; etc. }\end{array}$ & $\begin{array}{c}5 \\
(8,20 \%)\end{array}$ & $\begin{array}{c}19 \\
(31,15 \%)\end{array}$ & $\begin{array}{c}37 \\
(60,66 \%)\end{array}$ & \\
\hline $\begin{array}{l}\text { 5. Je vérifie que le style est approprié } \\
\text { et je veille à employer des connecteurs } \\
\text { adéquats. }\end{array}$ & $\begin{array}{c}2 \\
(3,28 \%)\end{array}$ & $\begin{array}{c}32 \\
(52,46 \%)\end{array}$ & $\begin{array}{c}27 \\
(44,26 \%)\end{array}$ & \\
\hline $\begin{array}{l}\text { 6. J'essaie de repérer les points à pro- } \\
\text { pos desquels je ne suis pas encore sûr. }\end{array}$ & $\begin{array}{c}2 \\
(3,28 \%)\end{array}$ & $\begin{array}{c}27 \\
(44,26 \%)\end{array}$ & $\begin{array}{c}32 \\
(52,46 \%)\end{array}$ & \\
\hline $\begin{array}{l}\text { 7. J'effectue les recherches et vérifica- } \\
\text { tions correspondantes. }\end{array}$ & $\begin{array}{c}3 \\
(4,92 \%)\end{array}$ & $\begin{array}{c}22 \\
(36,07 \%)\end{array}$ & $\begin{array}{c}36 \\
(59,02 \%)\end{array}$ & \\
\hline $\begin{array}{l}\text { 8. Je laisse mon texte de côté pendant } \\
\text { une journée pour le relire ensuite avec } \\
\text { un regard neuf. }\end{array}$ & $\begin{array}{c}21 \\
(34,43 \%)\end{array}$ & $\begin{array}{c}30 \\
(49,18 \%)\end{array}$ & $\begin{array}{c}10 \\
(16,39 \%)\end{array}$ & \\
\hline $\begin{array}{l}\text { 9. Je rédige une première version en } \\
\text { utilisant les stratégies de vérification } \\
\text { de production écrite. J'écris ensuite } \\
\text { une deuxième version }\end{array}$ & $\begin{array}{c}18 \\
(29,51 \%)\end{array}$ & $\begin{array}{c}33 \\
54,10 \%)\end{array}$ & $\begin{array}{c}10 \\
(16,39 \%)\end{array}$ & \\
\hline $\begin{array}{l}\text { 10. Je vérifie avoir bien compris les } \\
\text { consignes. }\end{array}$ & & $\begin{array}{c}22 \\
(36,07 \%)\end{array}$ & $\begin{array}{c}39 \\
(63,93 \%)\end{array}$ & \\
\hline
\end{tabular}


11. Je reviens en arrière pour vérifier si mes hypothèses initiales étaient correctes ou sensées ou bien s'il faut que je les réexamine.

12. Je prends connaissance de la tâche à effectuer et j'essaie de comprendre les consignes, par exemple : après avoir lu le texte, je dois rechercher la phrase clé de chaque paragraphe ; je dois essayer de penser aux questions que le professeur pourrait poser.

13. J'identifie les difficultés et réfléchis aux solutions possibles, par exemple : consulter le dictionnaire, demander à un camarade ou à un professeur.

14. Je réfléchis aux conditions de travail qui me conviennent le mieux pour effectuer cette tâche, par exemple : être dans un endroit tranquille.

15. Je décide sur quoi me concentrer, par exemple : lorsque je commence à rédiger, je ne me préoccupe pas si les temps sont corrects, je me contente de développer mes idées. Par la suite, je ferai une relecture pour corriger ce que j'aurais écrit. 16. Je ne me laisse pas abattre lorsque je retrouve des difficultés, par exemple : si je ne comprends pas la phrase clé d'un paragraphe, je passe au suivant pour voir si cela peut $m$ 'aider.

17. Je revérifie mes idées initiales.

18. Je vérifie l'efficacité de stratégies utilisées et je change ce qui est nécessaire, par exemple : lorsque je pensais pouvoir effectuer une tâche en lisant simplement le texte pour en saisir le sens général. Cependant, il faudrait que je relise le texte plus lentement et que je recherche la signification de certains mots clés. 19. Une fois que j'ai réussi à comprendre, je détermine ce sur quoi je vais continuer à me concentrer, par exemple : j'ai réussi à rédiger un paragraphe, un récit, une lettre simple, etc. mais il va maintenant falloir essayer d'employer un style plus élaboré.

$\begin{array}{ccc}6( & 28 & 27 \\ 9,84 \%) & (45,90 \%) & (44,26 \%) \\ & & \\ 10 & 33 & 18 \\ (16,39 \%) & (54,10 \%) & (29,51 \%)\end{array}$

17

$(27,87 \%) \quad(72,13 \%)$

$\begin{array}{lll}3 & 22 & 36\end{array}$

$(4,92 \%) \quad(36,07 \%) \quad(59,02 \%)$

$12 \quad 29$

20

$(19,67 \%) \quad(47,54 \%) \quad(32,79 \%)$

27

34

$(44,26 \%) \quad(55,74 \%)$

29

24

$\begin{array}{ccc}(13,11 \%) & (47,54 \%) & (39,34 \%) \\ 2 & 33 & 26 \\ (3,28 \%) & (54,10 \%) & (42,62 \%)\end{array}$

12

30

19

$(19,67 \%) \quad(49,18 \%) \quad(31,15 \%)$ 
20. J'identifie mes liens avec la francophonie dans ce que je lis, par exemple : je fais une liste des éléments culturels francophones et je compare avec ma vie. 21. Je crée des liens avec la francophonie dans ce que j'écris.
26

$(42,62 \%)$

26

$(42,62 \%)$

$(13,11 \%)$

$(1,64 \%)$

Par rapport aux stratégies métacognitives d'autorégulation, nous pouvons observer un pourcentage important d'utilisation pour la stratégie 1, dont 83\% des apprenants affirme l'utiliser souvent. Ensuite, 68,85\% des apprenants utilise souvent la stratégie 3. Finalement, autour d'un 60\% utilise souvent les stratégies 7 et 10 . Nous retrouvons des pourcentages un peu plus bas pour les stratégies 2 , 5 et 11 avec des pourcentages de 44,26\% d'apprenants utilisant souvent ces stratégies. Pour les stratégies 5, 9 et 18 plus d'un 50\% affirme utiliser quelquefois ces stratégies et presqu'un 50\% pour les stratégies 8 et 19 ainsi que 45,90\% pour la stratégie 17 . Il est aussi important de signaler que $34,43 \%$ et $29,51 \%$ des apprenants affirment ne jamais utiliser les stratégies 8 et 9 respectivement.

En ce qui concerne les stratégies d'autogestion 14 et 16, plus d'un 55\% emploie souvent ces stratégies. Pour la stratégie 12, nous remarquons qu'un 54\% des apprenants l'utilisent. Et pour les stratégies 15 et 16 , environ $45 \%$ affirme les utiliser quelquefois. Plus de $15 \%$ des étudiants, signalent ne jamais utiliser les stratégies 12 et 15.

Pour les stratégies d'attention, la question 20 présente des pourcentages identiques: $42.62 \%$ des apprenants utilisant quelquefois et $42.62 \%$, n'utilisant jamais cette stratégie, 62,30\% utilisant quelquefois la stratégie 21 et 32,79\% ne l'utilisant jamais. Pour la stratégie 6, nous observons qu'un 52,46\% des étudiants utilise souvent cette stratégie et 44,26 quelquefois.

Pour la stratégie 4 d'autorégulation et identification du problème, nous pouvons signaler que $60,66 \%$ des apprenants utilisent souvent cette stratégie et $31,15 \%$ l'utilisent quelquefois.

La stratégie 13 d’identification du problème présente un pourcentage important de $72 \%$ des apprenants disant l'utiliser souvent et 27,87 quelquefois.

Ces résultats confirment que les stratégies métacognitives d'autorégulation, d'autogestion, d'attention et d'identification du problème sont bien appréciées par la plupart des apprenants, ce qui pourrait s'expliquer par le désir de vérifier de façon individuelle, autonome et ponctuelle les connaissances acquises, ce qui n'assure pas nécessairement la reprise postérieure ou à long terme des mêmes connaissances. Nous pouvons aussi signaler que les stratégies d'attention concernant la mise en rapport des aspects linguistiques avec la culture francophone ne sont pas très communes chez les apprenants débutants. En effet, ils ont encore des difficultés pour identifier des éléments culturels francophones dans leurs lectures et pour créer des liens avec la francophonie dans leurs productions écrites. 


\subsection{Les stratégies socio-affectives}

Les stratégies socio-affectives impliquent l'interaction avec une autre personne, dans le but de favoriser l'apprentissage et le contrôle de la dimension affective accompagnant l'apprentissage. Le tableau ci-dessous illustre les différentes stratégies socioaffectives utilisées par les apprenants de la première année :

Tableau 7

Les stratégies socio-affectives

\begin{tabular}{|c|c|c|c|c|}
\hline Stratégies & Jamais & Quelquefois & Souvent & $\begin{array}{l}\text { Sans } \\
\text { réponse }\end{array}$ \\
\hline $\begin{array}{l}\text { 1. J'identifie ce qui me touche et les } \\
\text { émotions que je ressens à l'égard de } \\
\text { la tâche de lecture/écriture proposée. }\end{array}$ & $\begin{array}{c}7 \\
(11,48 \%)\end{array}$ & $\begin{array}{c}34 \\
(55,74 \%)\end{array}$ & $\begin{array}{c}20 \\
(32,79 \%)\end{array}$ & \\
\hline $\begin{array}{l}\text { 2. J'analyse mes émotions et je déter- } \\
\text { mine ce qu'elles révèlent à mon sujet (à } \\
\text { partir de mes expériences personnelles). }\end{array}$ & $\begin{array}{c}10 \\
(16,39 \%)\end{array}$ & $\begin{array}{c}31 \\
(50,82 \%)\end{array}$ & $\begin{array}{c}20 \\
(32,79 \%)\end{array}$ & \\
\hline $\begin{array}{l}\text { 3. Je décide ce que j'accepte et ce que } \\
\text { je rejette du texte. }\end{array}$ & $\begin{array}{c}7 \\
(11,48 \%)\end{array}$ & $\begin{array}{c}29 \\
(47,54 \%)\end{array}$ & $\begin{array}{c}25 \\
(40,98 \%)\end{array}$ & \\
\hline $\begin{array}{l}\text { 4. J'exprime la façon dont ce texte } \\
\text { m'affecte. }\end{array}$ & $\begin{array}{c}19 \\
(31,15 \%)\end{array}$ & $\begin{array}{c}33 \\
(54,10 \%)\end{array}$ & $\begin{array}{c}9( \\
14,75 \%)\end{array}$ & \\
\hline $\begin{array}{l}\text { 5. J'exprime la façon dont la rédac- } \\
\text { tion d'un texte m'affecte. }\end{array}$ & $\begin{array}{c}21 \\
(34,43 \%)\end{array}$ & $\begin{array}{c}27 \\
(44,26 \%)\end{array}$ & $\begin{array}{c}12 \\
(19,67 \%)\end{array}$ & $\begin{array}{c}1 \\
(1,64 \%)\end{array}$ \\
\hline $\begin{array}{l}\text { 6. J'adopte une attitude positive par } \\
\text { rapport à la tâche. }\end{array}$ & $\begin{array}{c}3 \\
(4,92 \%)\end{array}$ & $\begin{array}{c}28 \\
(45,90 \%)\end{array}$ & $\begin{array}{c}30 \\
(49,18 \%)\end{array}$ & \\
\hline $\begin{array}{l}\text { 7. J'accepte de faire une tâche malgré } \\
\text { mes incertitudes. }\end{array}$ & $\begin{array}{c}11 \\
(18,03 \%)\end{array}$ & $\begin{array}{c}29 \\
(47,54 \%)\end{array}$ & $\begin{array}{c}21 \\
(34,43 \%)\end{array}$ & \\
\hline $\begin{array}{l}\text { 8. J'identifie les raisons pour les- } \\
\text { quelles je fais des efforts pour réaliser } \\
\text { la tâche. }\end{array}$ & $\begin{array}{c}7 \\
(11,48 \%)\end{array}$ & $\begin{array}{c}32 \\
(52,46 \%)\end{array}$ & $\begin{array}{c}22 \\
(36,07 \%)\end{array}$ & \\
\hline 9. J'évalue mes chances de succès. & $\begin{array}{c}3 \\
(4,92 \%)\end{array}$ & $\begin{array}{c}29 \\
(47,54 \%)\end{array}$ & $\begin{array}{c}29 \\
(47,54 \%)\end{array}$ & \\
\hline $\begin{array}{l}\text { 10. J'envisage les choix à faire pour } \\
\text { assurer le bon déroulement de la tâche. }\end{array}$ & $\begin{array}{c}3 \\
(4,92 \%)\end{array}$ & $\begin{array}{c}42 \\
(68,85 \%)\end{array}$ & $\begin{array}{c}15 \\
(24,59 \%)\end{array}$ & $\begin{array}{c}1 \\
(1,64 \%)\end{array}$ \\
\hline $\begin{array}{l}\text { 11. Je persiste à bien travailler jusqu'à } \\
\text { la fin de la tâche }\end{array}$ & $3(4,92 \%)$ & $26(42,62 \%)$ & $\begin{array}{c}32 \\
(52,46 \%)\end{array}$ & \\
\hline $\begin{array}{l}\text { 12. Je prends des décisions pour aug- } \\
\text { menter ma motivation par rapport à } \\
\text { ce que je lis/écris. }\end{array}$ & $\begin{array}{c}5 \\
(8,20 \%)\end{array}$ & $\begin{array}{c}24 \\
(39,34 \%)\end{array}$ & $\begin{array}{c}32 \\
(52,46 \%)\end{array}$ & \\
\hline $\begin{array}{l}\text { 13. Je demande des précisions sur le } \\
\text { but et les consignes de la tâche. }\end{array}$ & $\begin{array}{c}3 \\
(4,92 \%)\end{array}$ & $\begin{array}{c}27 \\
(44,26 \%)\end{array}$ & $\begin{array}{c}31 \\
(50,82 \%)\end{array}$ & \\
\hline 14. Je demande de l'aide. & $\begin{array}{c}2 \\
(3,28 \%)\end{array}$ & $\begin{array}{c}31 \\
(50,82 \%)\end{array}$ & $\begin{array}{c}26 \\
(42,62 \%)\end{array}$ & $\begin{array}{c}2 \\
(3,28 \%)\end{array}$ \\
\hline
\end{tabular}


15. Je vérifie ma compréhension de la tâche en m'adressant au professeur ou à un camarade.

16. J'aide à planifier le travail d'équipe.

17. Je suis responsable de mon travail et d'aider les autres.

18. Je partage mes idées, mes moyens et mes ressources.

19. J'écoute les idées des autres et j'en tiens compte.

20. J'encourage et je félicite les membres de mon équipe.

21. J'évalue mon degré d'anxiété.

$(54,10 \%) \quad(37,70 \%)$

$2 \quad 20 \quad 39$

$(3,28 \%) \quad(32,79 \%) \quad(63,93 \%)$

$15-46$

$(24,59 \%) \quad(75,41 \%)$

$\begin{array}{lll}2 & 20 & 39\end{array}$

$(3,28 \%) \quad(32,79 \%) \quad(63,93 \%)$

$2 \quad 11 \quad 48$

$(3,28 \%) \quad(18,03 \%) \quad(78,69 \%)$

$\begin{array}{lll}1 & 18 & 42\end{array}$

$(1,64 \%) \quad(29,51 \%) \quad(68,85 \%)$

$\begin{array}{lll}9 & 32 & 20\end{array}$

$(14,75 \%) \quad(52,46 \%) \quad(32,79 \%)$

22. J'utilise diverses techniques en

13

20

28

vue de réduire mon anxiété, par exemple : respiration, activité de dé-

$(21,31 \%) \quad(32,79 \%) \quad(45,90 \%)$
tente, encouragement, visualisation positive, division de la tâche en petite partie pour réduire sa complexité.

23. Je pense à ce que j'ai déjà réussi.

24. Je me centre sur mes points forts.

\begin{tabular}{cccc}
$(14,75 \%)$ & $(40,98 \%)$ & $(44,26 \%)$ & \\
1 & 20 & 40 & \\
$(1,64 \%)$ & $(32,79 \%)$ & $(65,57 \%)$ & \\
2 & 20 & 39 & \\
$(3,28 \%)$ & $(32,79 \%)$ & $(63,93 \%)$ & \\
5 & 35 & 19 & 2 \\
$(8,20 \%)$ & $(57,38 \%)$ & $(31,15 \%)$ & $(3,28 \%)$ \\
17 & 36 & 8 & \\
$(27,87 \%)$ & $(59,02 \%)$ & $(13,11 \%)$ & \\
16 & 27 & 18 & \\
$(26,23 \%)$ & $(44,26 \%)$ & $(29,51 \%)$ & \\
& \multicolumn{3}{r}{}
\end{tabular}

25. Je me concentre sur mes chances de succès.

26. Je ne capitule pas devant la difficulté et je n'essaie pas de deviner « à la sauvage ».

27. Je demande à un (e) ami (e) de m'interroger.

28. Je demande à un ami, à un autre camarade ou à un professeur de lire mon travail.

$(26,23 \%)$

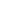

$26 \%$$$
40
$$

, $93 \%$

$31,15 \%)$

8

$(29,51 \%)$

La stratégie de gestion des émotions est révélée par les énoncés $1,2,3,4,5$, $8,10,11,21,22,23,24$ et 25 . Elles sont souvent ou quelquefois utilisées par la plupart des apprenants. Nous noterons toutefois qu'un nombre important d'étudiants n'utilise jamais les stratégies 4 et 5 .

La stratégie de coopération est représentée par les questions 16, 17, 18, 19 et 20. Elle est souvent employée par plus d'un $60 \%$ des apprenants. Nonobstant, pour les stratégies 27 et 28 , nous observons que plus d'un $40 \%$ s'en sert quelquefois et plus d'un $20 \%$ ne les utilise jamais.

La stratégie de clarification et vérification des énoncés 13,14 et 15 est souvent ou quelquefois utilisée par plus d'un $40 \%$ des apprenants. 
Les stratégies d'autorenforcement 6, 9, 12 et 26 sont souvent ou quelquefois utilisées par une grande partie des apprenants.

Finalement, par rapport à la stratégie de gestion des émotions et d'autorenforcement 7 , nous apercevons que plus d'un $40 \%$ l'utilise quelquefois, plus d'un $30 \%$ l'emploie souvent et moins d'un $20 \%$ ne l'utilise jamais.

Pour résumer, il semble que les apprenants de la première année emploient souvent ou quelquefois les stratégies socio-affectives lorsqu'ils en ont besoin, mais il ne faudrait pas négliger qu'un nombre important d'apprenants ne se sert jamais des stratégies de coopération représentées par les questions 27 et 28 . Cette stratégie concerne surtout la demande d'aide à un ami, à un camarade ou à un professeur. Cette situation attire notre attention, car pour l'apprentissage d'une langue étrangère, il est très important et commun de demander de l'aide ou des conseils à autre personne pour pouvoir améliorer et pratiquer la langue.

Il est pertinent de signaler aussi qu'un nombre important d'étudiants n'utilise jamais les stratégies de gestion des émotions, représentées par les questions 4 et 5 concernant les sentiments éprouvés par les apprenants lors de la lecture ou la rédaction d'un texte.

\section{Conclusions}

En guise de conclusion, nous pouvons affirmer que les apprenants de la première année des filières d'enseignement du français et de français de l'Université du Costa Rica emploient différentes stratégies cognitives. Parmi les plus remarquables, nous retrouvons les stratégies d'inférence, de déduction, de traduction et de répétition, actions qui correspondent au comportement espéré des apprenants débutants, voulant comprendre, fixer les règles et établir des comparaisons avec la L1 pour ainsi saisir le fonctionnement de la langue cible. Toutefois, les stratégies cognitives impliquant l'aspect ludique semblent ne pas être très appréciées. Nous pouvons expliquer cela par le fait que dans la culture costaricienne, les activités ludiques pour mémoriser le lexique ou les règles grammaticales ne sont pas très courantes en classe de langue.

Il est important de souligner que bien qu'il s'agisse d'un groupe d'apprenants débutants, ils utilisent souvent des stratégies métacognitives impliquant l'autorégulation et l'autogestion. Cette caractéristique révèle une certaine autonomie, leur permettant d'acquérir, stocker, retrouver et utiliser les informations indispensables pour rendre leur apprentissage et acquisition de la L2 plus efficace, plus agréable et plus transférable vers de nouvelles situations. Néanmoins, nous constatons d'une part que quelques stratégies d'autorégulation ne sont pas très utilisées, surtout celles liées aux activités impliquant la révision exhaustive des tâches. D'autre part, les stratégies d'attention visant à associer des éléments linguistiques à des aspects culturels de la langue cible sont peu employées, étant donné qu'il s'agit des étudiants débutants n'ayant pas encore trop connaissances assimilées en ce qui concerne le monde francophone. 
Quant aux stratégies socio-affectives, nous remarquons que les stratégies de gestions des émotions, de clarification et de vérification et d'autorenforcement sont très utilisées, attitude justifiée par leur intérêt et leur motivation vis-à-vis la maîtrise de la langue cible. Cependant, la stratégie de coopération impliquant le travail avec des camarades est beaucoup moins utilisée, car les étudiants costariciens ne sont pas habitués en général à travailler de manière collaborative.

\section{Bibliographie}

Atlan, J. (2000). « L'utilisation des stratégies d'apprentissage d'une langue dans un environnement des TICE », ALSIC, 3, 1. pp. 109- 123.

Bégin, C. (2008). "Les stratégies d'apprentissage : un cadre de référence simplifié ». Revue de sciences de l'éducation, 34 (1), pp. 47-67.

Boudon, M. (2006). Les stratégies d'apprentissage du Français Langue Étrangère (FLE) des étudiants coréens à l'université en Corée. 프랑스문화연구, 12, pp.311-345.

Cyr, P. (1998). Les stratégies d'apprentissage. Paris : CLE International.

Guan, X. (2005). L'entraînement à l'utilisation des stratégies d'écoute-vers un enseignement plus efficace de la compréhension orale en L2. Master 2 professionnel: Université Lumière Lyon 2.

Harris, V. (2002). Aider les apprenants à apprendre : à la recherche de stratégies d'enseignement et d'apprentissage dans les classes de langue en Europe. Strasbourg, Conseil de l'Europe.

Lafontaine, M. (2005). «Stratégies d'apprentissage et réussite en anglais langue seconde chez des élèves de $5^{\mathrm{e}}$ secondaire : vers une validation de la typologie d'O'Malley et Chamot ». Langues et linguistique, 31, pp. 111-143.

Larue, C. et R. Cossette (2005). Stratégies d'apprentissage et apprentissage par problèmes. Montréal, Cégep du Vieux Montréal.

Lee, P. (2011). Stratégies d'apprentissage des langues secondes dans un environnement informatisé : une méta-analyse qualitative de l'utilisation du courrier électronique. Thèse doctorale en philosophie : Dalhousie University.

MacIntyre, P.D. (1994). "Toward a social psychological model of strategy use» Foreign Language Annals, 27, 2. pp. 185-195.

O'Malley, J.M. et Chamot, A.U. (1990). Learning strategies in second language acquisition. Cambridge: Cambridge University Press.

Tang, W.J. (2011). Les stratégies d'apprentissage d'un groupe cosmopolite d'apprenants d'anglais langue étrangère dans un monde virtuel-Second Life. Mémoire de master 2 : Université Stendhal.

Wenden, A L. (1987). "Conceptual background and utility.". In Wenden A. L. et Rubin J. (dirs.). Learner Strategies in Language Learning. Hemel, Hempstead : Prentice Hall International. pp. 3-13. 


\section{Anexe 1}

Universidad de Costa Rica

Escuela de Lenguas Modernas

Proyecto de Investigación

"Estudio sobre el uso de las estrategias de aprendizaje del estudiantado de las carreras de Bachillerato en Francés y Bachillerato en Enseñanza del Francés"

Objectif: Connaître les stratégies d'apprentissage des apprenants des filières « Bachillerato en Francés » et « Bachillerato en Enseñanza del Francés ».

\section{A. Information personnelle}

1. Sexe :

$\mathrm{H}$

2. Age :

18-20

21-25

26-30

plus de 31

3. Situation de

célibataire

famille :

marié(e)

séparé (e)

divorcé (e)

autres:

4. Vous avez des

$\square \quad$ oui

enfants :

5. Lieu de

San José

provenance :

$\begin{array}{ll}\square & \text { San José } \\ \square & \text { Heredia } \\ \square & \text { Alajuela } \\ \square & \text { Limón } \\ \square & \text { Puntarenas } \\ \square & \text { Guanacaste } \\ \square & \text { Cartago }\end{array}$

6. Vous avez fait vos études secondaires dans un lycée :

Public

Privé

Semi-privé

7. Vous avez suivi vos études secondaires dans un lycée :

Du jour

Nocturne 
8. Vous avez suivi vos études secondaires dans un lycée :

Académique

Technique

9. Filières : $\quad \square \quad$ Bachillerato en Francés

10. Année : $\quad \square \quad$ Première année

$\square \quad$ Deuxième année

Troisième année

Quatrième année

11. C'est la première fois que vous suivez le cours de ce semestre :

oui

non

12. Vous avez actuellement une bourse d'études :

oui

non

13. Vous avez obtenu votre bourse dû :

à votre niveau socioéconomique

à votre excellence académique

à la participation aux activités sportives ou culturelles aux heures d'assistanat

14. Actuellement,

oui vous travaillez :

non

15. Si vous travaillez, vous travaillez : moins de 20 heures par semaine plus de 20 heures par semaine

16. Vous suivez d'autres études :

oui - Dans quelle filière?

non

17. Vous avez choisi les filières de français :

en première option

en deuxième option

en troisième option

18. Vous vous sentez à l'aise dans les filières de français :

oui

non 
19. Combien d'heures par semaine vous consacrez à vos études des cours de français :

moins de 5 heures par semaine

6 à 10 heures par semaine

11 à 15 heures par semaine

16 à 20 heures par semaine

plus de 20 heures par semaine

20. Vous suivez tous les cours correspondant à votre année universitaire :

oui

non

21. Vous assistez régulièrement aux cours :

oui

non

presque tout le temps

22. Vous parlez d'autres langues (à part le français et l'espagnol) :

oui - Lesquelles?

non

B. Répondez aux questions suivantes. Cochez une seule option.

Les stratégies de compréhension écrite

\section{Jamais Quelquefois Souvent ou} très souvent

Avant la lecture

1. Je vérifie avoir bien compris les consignes.

2. J'examine la typographie (le titre, les images, etc.) du document afin de déterminer le type de texte, par exemple : lettre, poème, article, etc.

3. Je regarde attentivement les images et le titre pour pouvoir déduire le sujet en question.

4. J'essaie de me remémorer autant de mots que possible en rapport avec le sujet abordé.

Pendant la lecture

1. Je lis la totalité du texte afin d'en saisir l'idée générale.

2. Je repère les mots apparentés ou qui me sont familiers. 
3. J'identifie les termes qui me paraissent être des mots-clés.

4. Je m'appuie sur mes connaissances acquises pour en déduire des hypothèses plausibles.

5. J'essaie de tirer des indices à partir de la ponctuation.

6. Je prononce à haute voix les mots qui ne me sont pas familiers dans le but de mieux les comprendre.

7. Je lis le texte à haute voix pour repérer la composition des phrases et les parties sur lesquelles je dois me concentrer les unes après les autres.

8. Je substitue les mots que je ne connais pas par d'autres mots dans ma langue maternelle.

9. Je décompose les mots qui ne me sont pas familiers pour associer certains éléments à des mots que je connais.

10. Je repère les mots nouveaux et je cherche leur sens dans le dictionnaire.

11. Je ne capitule pas devant la difficulté et je n'essaie pas de deviner « à la sauvage ».

12. Je regarde attentivement la terminaison des verbes pour en déterminer le temps et identifier le sujet.

$$
\text { Après la lecture }
$$

1. Je reviens en arrière pour vérifier si mes hypothèses initiales étaient correctes ou sensées ou bien s'il faut que je les réexamine.

2. Je fais un résumé de la lecture.

\section{Les stratégies de mémorisation du vocabulaire}

\section{Jamais Quelquefois Souvent ou}

très souvent

1. J'associe un mot que j'essaie d'apprendre un mot similaire dans ma langue maternelle.

2. J'en fais un dessin.

3. J'écris les mots plusieurs fois de suite.

4. J'utilise la technique « lire-cacher-tester-vérifier ».

5. Je regarde le mot, puis je ferme les yeux et j'essaie de me le représenter dans mon esprit (mémoire photographique).

6. Je décompose les mots longs.

7. J'utilise les mots pour en faire un poème de toute sorte. 
8. Je mets tout autour de ma chambre des notes autocollantes sur lesquelles j'ai inscrit les mots à apprendre.

9. Je crée deux jeux de cartes - l'un contenant les mots en langue étrangère, l'autre les images ou les mots correspondants dans ma langue maternelle - afin d'apprendre de façon ludique.

10. Je sépare les mots de leurs articles et j'essaie ensuite de les rapparier.

11. Je fais une recherche sur le mot et je l'utilise dans des phrases.

12. Je crée un exercice à trous en y intégrant les mots nouveaux.

13. Je prononce les mots à voix haute plusieurs fois de suite.

14. Je demande à un (e) ami (e) de m'interroger.

15. Je fais des exercices en ligne (exercices à trous, question à choix multiple, etc.) pour assimiler le vocabulaire.

16. Je regroupe les mots en familles.

17. Je repère dans des documents (écrits, sonores, visuels) le vocabulaire étudié pour le comprendre en contexte afin de le fixer dans ma mémoire.

18. Je crée un réseau d'associations, par exemple :

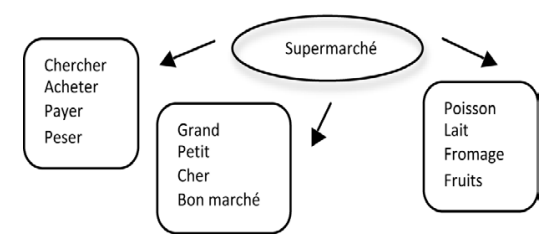

\section{Les stratégies de mémorisation des règles grammaticales}

\section{Jamais Quelquefois Souvent ou très souvent}

1. Je mets les règles en musique.

2. Je fais des jeux, par exemple : classer les différents groupes de verbes.

3. Je crée un mot en utilisant les premières lettres, par exemple : ANDATE pour le passé composé.

4. J'utilise des images.

5. Je mets tout autour de ma chambre des notes autocollantes sur lesquelles j'ai inscrit les verbes ou les règles grammaticales à apprendre. 
6. Je crée un réseau d'associations, par exemple :

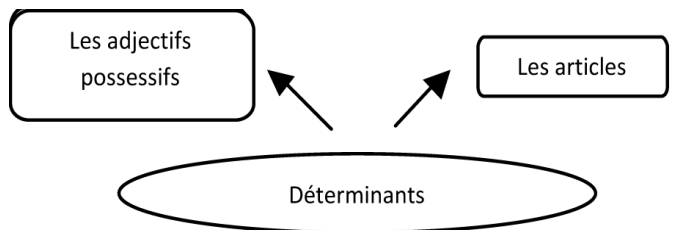

7. Je me base sur les similitudes et les différences qui existent avec ma langue maternelle.

8. Je suis attentif à la présence de règles, par exemple : je souligne ou entoure les exemples correspondant à une règle grammaticale dans un document.

9. J'écris plusieurs fois les règles.

10. Je répète oralement les règles.

\section{Les stratégies d'écriture}

\section{Jamais Quelquefois Souvent ou très souvent}

\section{Rassembler des informations}

1. Je réfléchis aux exigences posées par tâches et rassemble toutes les idées que je veux exprimer.

2. Je réunis des informations supplémentaires en lisant, en parlant avec d'autres personnes et en me remémorant les mots et expressions acquis que je pourrais utiliser dans le cadre de cette tâche.

$$
\text { Développer des idées }
$$

1. Je dresse une liste d'idées clés.

2. Je choisis la façon d'organiser et de communiquer mes idées (hiérarchisation, classement, argumentation, exemples).

3. Je fais un plan de travail pour indiquer l'ordre de présentation des idées.

\section{Travail de rédaction}

1. Je suis le plan établi préalablement.

2. Je rajoute des idées au fur et à mesure que je rédige.

3. Je consulte le dictionnaire lorsque je ne connais pas un mot ou une expression dont j'ai besoin.

4. J'essaie de trouver une autre façon d'exprimer le mot ou l'expression que je ne connais pas.

5. J'essaie d'être aussi précis que possible en employant l'adjectif, le verbe, le nom exacts. 
6. J'utilise des connecteurs précis tels que « tout d'abord, puis, ensuite, enfin, etc. ».

7. J'essaie d'employer un style plus élaboré en recourant au lexique approprié.

8. J'essaie d'employer un style plus élaboré en faisant appel aux règles de grammaire que $\mathrm{j}$ 'ai apprises.

9. Je veille à être précis.

Vérification de la production écrite

1. Je lis la totalité du texte pour voir s'il est cohérent.

2. Je lis séparément chacun des mots pour voir s'ils ont l'air correct.

3. Je prononce les mots pour moi-même afin de voir s'ils sonnent bien.

4. Je relis mon travail en faisant attention à mes fautes habituelles de grammaire telles que : l'accord des adjectifs, des verbes; les conjugaisons des verbes; etc.

5. Je vérifie que le style est approprié et je veille à employer des connecteurs adéquats.

6. J'essaie de repérer les points à propos desquels je ne suis pas encore sûr.

7. J'effectue les recherches et vérifications correspondantes.

8. Je laisse mon texte de côté pendant une journée pour le relire ensuite avec un regard neuf.

\section{Evaluation de mon travail}

1. Je rédige une première version en utilisant les stratégies de vérification de production écrite. J'écris ensuite une deuxième version. 2. Je demande à un ami, à un autre camarade ou à un professeur de lire mon travail.

3. Je rédige la version finale.

\section{Les stratégies métacognitives}

\begin{tabular}{ccc}
\hline Jamais & Quelquefois & $\begin{array}{c}\text { Souvent ou } \\
\text { très souvent }\end{array}$ \\
\hline
\end{tabular}

1. Je prends connaissance de la tâche à effectuer et j'essaie de comprendre les consignes, par exemple : après avoir lu le texte, je dois rechercher la phrase clé de chaque paragraphe; je dois essayer de penser aux questions que le professeur pourrait poser.

2. J'identifie les difficultés et réfléchis aux solutions possibles, par exemple : consulter le dictionnaire, demander à un camarade ou à un professeur. 
3. Je réfléchis aux conditions de travail qui me conviennent le mieux pour effectuer cette tâche, par exemple : être dans un endroit tranquille.

4. Je décide sur quoi me concentrer, par exemple : lorsque je commence à rédiger, je ne me préoccupe pas si les temps sont corrects, je me contente de développer mes idées. Par la suite, je ferai une relecture pour corriger ce que j'aurais écrit.

5. Je ne me laisse pas abattre lorsque je retrouve des difficultés, par exemple : si je ne comprends pas la phrase clé d'un paragraphe, je passe au suivant pour voir si cela peut m'aider.

6. Je revérifie mes idées initiales.

7. Je vérifie l'efficacité de stratégies utilisées et je change ce qui est nécessaire, par exemple : lorsque je pensais pouvoir effectuer une tâche en lisant simplement le texte pour en saisir le sens général. Cependant, il faudrait que je relise le texte plus lentement et que je recherche la signification de certains mots clés.

8. Une fois que j'ai réussi à comprendre, je détermine ce sur quoi je vais continuer à me concentrer, par exemple : j'ai réussi à rédiger un paragraphe, un récit, une lettre simple, etc. mais il va maintenant falloir essayer d'employer un style plus élaboré.

\section{Les stratégies socio-affectives}

\section{Jamais Quelquefois Souvent ou très souvent}

1. J'identifie ce qui me touche et les émotions que je ressens à l'égard de la tâche de lecture/écriture proposée.

2. J'analyse mes émotions et je détermine ce qu'elles révèlent à mon sujet (à partir de mes expériences personnelles).

3. J'identifie mes liens avec la francophonie dans ce que je lis, par exemple : je fais une liste des éléments culturels francophones et je compare avec ma vie.

4. Je crée des liens avec la francophonie dans ce que j'écris.

5. Je décide ce que j'accepte et ce que je rejette du texte.

6. J'exprime la façon dont ce texte m'affecte.

7. J'exprime la façon dont la rédaction d'un texte m'affecte.

8. J'adopte une attitude positive par rapport à la tâche. 
9. J'accepte de faire une tâche malgré mes incertitudes.

10. J'identifie les raisons pour lesquelles je fais des efforts pour réaliser la tâche.

11. J'évalue mes chances de succès.

12. J'envisage les choix à faire pour assurer le bon déroulement de la tâche.

13. Je persiste à bien travailler jusqu'à la fin de la tâche.

14. Je prends des décisions pour augmenter ma motivation par rapport à ce que je lis/écris.

15. Je demande des précisions sur le but et les consignes de la tâche.

16. Je demande de l'aide.

17. Je vérifie ma compréhension de la tâche en m'adressant au professeur ou à un camarade.

18. J'aide à planifier le travail d'équipe.

19. Je suis responsable de mon travail et d'aider les autres.

20. Je partage mes idées, mes moyens et mes ressources.

21. J'écoute les idées des autres et j'en tiens compte.

22. J'encourage et je félicite les membres de mon équipe.

23. J'évalue mon degré d'anxiété.

24. J'utilise diverses techniques en vue de réduire mon anxiété, par exemple : respiration, activité de détente, encouragement, visualisation positive, division de la tâche en petite partie pour réduire sa complexité.

25. Je pense à ce que j’ai déjà réussi.

26. Je me centre sur mes points forts.

27. Je me concentre sur mes chances de succès. 
